Estudios sobre el Mensaje Periodístico ISSN-e: 1988-2696

http://dx.doi.org/10.5209/ESMP.55600

\title{
Análisis de los significados generados por el discurso de dos periódicos regionales sobre niños, niñas y adolescentes afectados por el conflicto armado colombiano
}

\author{
Ledis Bohórquez Farfán ${ }^{1}$; Giovanni Bohórquez-Pereira ${ }^{2}$; Nelson Molina Valencia ${ }^{3}$
}

Recibido: 13 de octubre de 2015 / Aceptado: 29 de abril de 2016

Resumen. El artículo presenta una investigación realizada con los periódicos regionales Vanguardia Liberal y El Frente bajo la pregunta por las categorías discursivas presentes acerca de niños afectados por el conflicto político armado del país. La revisión teórica confirma pocos estudios en este campo y la necesidad de este trabajo a partir de una comprensión de la psicología social discursiva cuya premisa es la construcción social de la realidad. El método seguido fue el análisis de contenido de 56 notas de periódicos seleccionadas mediante la técnica se semana compuesta. Los resultados indican que las categorías discursivas de los medios construyen hechos referidos a situaciones en los se ven involucrados los menores y se omite la voz de estos protagonistas. También se encontró el uso de categorías sociodemográficas en la construcción de condiciones estereotípicas empleadas para la generalización.

Palabras clave: Discurso; niños; Colombia; prensa; análisis de contenido.

\section{[en] Analysis of the meanings generated by the discourse of two regional newspapers on children and adolescents affected by the Colombian armed conflict}

\begin{abstract}
The article presents research carried out with regional newspaper Vanguardia Liberal and El Frente under the question on current discursive categories about children affected by the political conflict of the country. The theoretical review confirms few studies in this field as well as the need of this work based on a comprehension of discursive social psychology whose premise is the social construction of reality, and the effects rhetoric has on relationships. The method followed was the analysis of contents of 56 newspapers notes selected using the technique is made week. The results indicate that discursive categories of media build facts referred to situations in children see them involved and omitted the voice of these actors. The use of socio-demographic categories was also found in the construction of stereotypical terms employed for the generalization.
\end{abstract}

Keywords: Discourse; childs; Colombia; media; content analysis.

Sumario. 1. Introducción. 2. Estado de la cuestión; 2.1. Discursos sobre menores en medios impresos. Afectaciones de niños y niñas en contextos de conflicto armado. 3. Metodología; 3.1. Muestra: unidades de Análisis; 3.2. Procedimiento. 4. Resultados. 5. Discusión y conclusiones. 6. Referencias bibliográficas.

1 Universidad Pontificia Bolivariana, Seccional Bucaramanga (Colombia)

E-mail: ledis.bohorquez@ub.edu.co

2 Universidad Pontificia Bolivariana, Seccional Bucaramanga (Colombia)

E-mail: giovanni.bohorquez@upb.edu.co

3 Universidad del Valle (Colombia)

E-mail: nelson.molina@correounivalle.edu.co 
Cómo citar: Bohórquez Farfán, Ledis; Bohórquez-Pereira, Giovanni; y Molina Valencia, Nelson (2017): “Análisis de los significados generados por el discurso de dos periódicos regionales sobre niños, niñas y adolescentes afectados por el conflicto armado colombiano", en Estudios sobre el Mensaje Periodístico 23 (1), 343-358.

\section{Introducción}

El lenguaje de los medios tiene un efecto (corto plazo) y un impacto (largo plazo) en la sociedad actual. Está sustentado desde diversos puntos de vista cómo los medios de comunicación - "mass media"-, influyen en una sociedad y definen posiciones a su interior, mucho más en el contexto de un conflicto armado como el colombiano. En esta medida el lenguaje de los medios puede exacerbar e intensificar los alientos de la población hacia la paz o hacia la guerra. El manejo que dan a las noticias que involucran niños y niñas puede contribuir a movilizar a una sociedad en apoyo y solidaridad hacia ellos o por el contrario a rechazarlos y quizás la prensa, sin proponérselo, interviene en procesos de revictimización cuando los niños han sido afectados por la confrontación armada. Los periodistas tiene en sus manos una responsabilidad en el manejo de la información que como dice Albert Chillón Asensio citado por Correa (2008) los silencios, las exclamaciones y los interrogantes, no sólo son sustantivos sino también verbos y adjetivos que forman un recurso expresivo humano que favorecen la representación del pensamiento pero dejan ver que "....siempre existe una zona de claroscuro inevitable entre las palabras y su sentido..." (Correa, 2008: 2)

En este contexto y a partir de la preocupación en el tema, surgió la investigación Discurso en medios de comunicación impresos sobre niños afectados por el conflicto político armado colombiano, que tuvo como objetivo identificar las categorías discursivas por medio de las cuales dos periódicos regionales de Colombia (Vanguardia Liberal y El Frente), se refieren a los niños afectados por el conflicto político armado en Colombia. Un asunto relevante dada la situación de expresa vulnerabilidad de los niños y niñas que han sido afectados por el conflicto armado colombiano.

De acuerdo con la Fundación Agencia de Comunicaciones Periodismo Aliado de la Niñez, el Desarrollo Social y la Investigación - PANDI-, ha habido un cambio significativo en el manejo que los medios hacen en el tratamiento de las noticias relacionadas con la niñez, lo cual se ha notado en las consecuencias de las denuncias hechas: "Muchas veces nos preguntamos ¿de qué le sirve a una niña de nueve años hablar cuando es agredida sexualmente y contarlo ante el juez si la Suprema Corte dice que ese "manoseo" solo es injuria? ¿De qué le sirve a un bebé llorar de dolor si es su padre el que lo está matando? En estos dos casos les sirvió porque los medios de comunicación hicieron que su voz fuera escuchada por los políticos, por los jueces y por la sociedad. Los fallos cambiaron y los agresores purgan penas por sus delitos" (Agencia Pandi, 2008: 6)

\section{Estado de la cuestión. Afectaciones de niños y niñas en contextos de conflicto armado}

Las cifras demuestran que los niños y niñas son uno de los actores con mayores niveles de afectación por el conflicto armado, al punto que el Fondo de Naciones Unidas 
para la Infancia - UNICEF - declaró el año 2014 como un año devastador par a la infancia revelando que 15 millones de niños se encuentran atrapados en graves conflictos. "Ha habido niños asesinados mientras estudiaban en el aula y mientras dormían en sus camas; niños que han quedado huérfanos o que han sido secuestrados, torturados, reclutados, violados e incluso vendidos como esclavos. Nunca en la historia reciente tantos niños fueron sometidos a este tipo de indescriptible brutalidad". (UNICEF, 2014: Web)

En Colombia, "sesenta años de conflicto armado no solo han dejado una cifra aterradora de más de 2 millones de niños víctimas de masacres, secuestros, asesinatos, desplazamiento y desapariciones forzadas, sino que han obligado a varias generaciones a crecer a pesar del dolor de sus recuerdos. Decenas de colombianos vieron a muy temprana edad cómo los grupos armados torturaban a sus familiares o tuvieron que vivir sin sus padres porque la guerra se los había arrebatado." (Semana.com, 2015: web). "Durante 2013, se registraron 2.935 eventos asociados con conflicto armado y 851 eventos que implican afectaciones directas a niños, niñas y adolescentes. (Coalición contra la Vinculación de niños, niñas y jóvenes al conflicto armado en Colombia COALICO, 2013: web).

A pesar de las evidencias de los altos niveles de afectación que tienen los conflictos armados en los niños y niñas, no fue hasta la década de los noventa del pasado siglo y gracias a las consultas realizadas por UNICEF — en 1996- y otras organizaciones internacionales, que el mundo comenzó a prestar especial atención al impacto de la guerra en los niños. La situación ha sido tan grave que el Consejo de Seguridad de Naciones Unidas en el año 2005 estableció 6 graves violaciones a derechos de los niños y niñas en contextos de conflicto armado. Tales son: muerte o mutilación de niños, reclutamiento o utilización de niños por las fuerzas armadas estatales o grupos armados, ataques contra escuelas y hospitales, violación u otros abusos sexuales contra los niños y las niñas, el secuestro, y la negación de acceso a la asistencia humanitaria de niños y niñas. (ONU - Los Niños y los Conflictos Armados, 2013: web). Este tipo de acciones se establecen con el fin de promover la protección de los niños en situaciones de conflicto armado y poner fin a la impunidad de quienes cometen actos de violencia contra ellos. Todas las violaciones establecidas por la ONU se producen en Colombia, a las que COALICO (2013) ha incorporado una más: el desplazamiento forzado.

El asesinato o la mutilación son dos de los efectos comunes de la guerra en los niños y las niñas que viola el derecho a la vida e integridad personal. La ONU Consejo de Seguridad (2013) reporta que para Colombia en el año 2012, 52 niños (32 varones y 20 niñas) resultaron heridos, y 13 más (12 varones y 1 niña) muertos por explosión de minas antipersonas o restos explosivos. (ONU - Consejo de Seguridad, 2013). Para COALICO (2013) las cifras señalan 169 situaciones que atentaron contra el derecho a la vida e integridad personal de por lo menos 987 niños, niñas y adolescentes. “...Cifras del Centro de Memoria Histórica estiman que en 207 masacres ocurridas entre 1980 y 2010 fueron asesinados 469 niños y jóvenes. "Muchos de ellos fueron decapitados, arrojados a los ríos o enterrados en alguna fosa común". (El Colombiano.com, 2013: web)

Por su parte el reclutamiento de niños es una constante de los actores armados en contienda y una de las afectaciones más comunes en contextos de conflicto armado. En Colombia en 2012 se registraron 128 eventos que involucraron a 531 Niños, Niñas y Adolescentes, de los cuales 73 eran menores de 15 años al momento del reclu- 
tamiento, los y las demás (458) se encontraban entre los 8 y los 14 años (COALICO, 2013: web). Al respecto, la ONU - Consejo de Seguridad, 2013: web) concluye que los grupos armados ilegales seguían atacando y amenazando a los maestros que obstaculizaban el reclutamiento de niños, situación que evidencia lo difícil de la protección de los Derechos Humanos. "Desde el 19 de noviembre de 1999 hasta el 28 de febrero de 2013 fueron atendidos 5.120 niños, niñas y adolescentes que se desvincularon de los grupos ilegales. De estos, el 83\% (4.213) se entregó de manera voluntaria y el 17 por ciento (880) han sido recuperados por las fuerzas militares. E1 72 por ciento son hombres y el resto mujeres." (El Colombiano.com, 2013: web)

En cuanto a la categoría de ataque a escuelas y hospitales, dentro de las estadísticas del conflicto armado del Centro de Memoria Histórica en el informe Basta Ya, reporta que entre 1988 y 2012 se presentaron 5.138 casos de ataques a bienes civiles, dentro de los que se cuentan ataques a escuelas y hospitales. El Secretario General de Naciones Unidas sobre los niños y el conflicto armado en Colombia presentó en 2012 un informe en el que expone de qué manera en medio del conflicto se ha atacado tanto escuelas como hospitales afectando a niños y niñas. Por ejemplo en febrero de 2010, en el departamento de Nariño los miembros de las FARC-EP entraron por la fuerza a una escuela rural, donde una comunidad indígena celebraba una reunión. Posteriormente, por ejemplo, miembros de las Fuerzas Militares de Colombia atacaron a las FARC-EP en el interior de la escuela, lo que tuvo como consecuencia el desplazamiento de 300 miembros de la comunidad indígena, de quienes ambas partes sospechaban que apoyaban al otro bando" (ONU - Consejo de Seguridad, 2012: web).

Aunque es una constante, la violencia sexual es casi invisible más aún en contextos de guerra. La violencia sexual es una acción instrumentalizada como estrategia de guerra que humilla al contrincante y lo debilita desde lo más íntimo y privado.

Como arma de guerra, la violación se utiliza estratégica y tácticamente para alcanzar objetivos específicos en muchas formas de conflicto. Se utiliza para conquistar, expulsar o controlar a las mujeres y sus comunidades en tiempo de guerra o de conflicto interno. Como forma de tortura basada en el género, se utiliza para obtener información, castigar, intimidar y humillar [...] Se ataca a las mujeres para destruir su integridad física y psíquica. Se las ataca públicamente para poner de manifiesto la incapacidad de los varones para defender a la comunidad. Y se las ataca como portadoras de la siguiente generación: se destruye su capacidad reproductiva o se la utiliza dejándolas embarazadas por la fuerza de hijos del enemigo. (Amnistía Internacional, 2004: web).

En Colombia durante 2013 se identificaron 530 menores de edad víctimas de violencia sexual por actores armados, legales e ilegales. De ellas 260 estuvieron en situaciones de violación o intento de violación, 136 frente a abuso sexual y 134 en relación con la explotación y esclavitud sexual. (COALICO, 2013: web)

El secuestro es otra de las violaciones a la integridad personal, no obstante también se cuenta asociado a éste, el trabajo forzado, la esclavitud sexual y el reclutamiento. "En esta categoría se identificaron para 2013, tres tipos de hechos, el primero de control social que se evidenció solamente un caso en el ejercicio de monitoreo, correspondiente al departamento del Putumayo (Puerto Asís), 6 casos de secuestro 
que implicaron a 7 niños, niñas y adolescentes, y detenciones arbitrarias por parte del Ejército en las denominadas batidas, en las que se registraron 2.179 víctimas en 13 eventos..." (COALICO, 2013, pág. 4. Web).

De otro lado, razones políticas, de seguridad y de bloqueo contra algún actor armado son aducidas por quienes en medio del conflicto impiden el acceso de la ayuda humanitaria que afecta a la población civil en general, entre la que se cuentan niños y niñas. En Colombia esta situación sigue presentándose debido a la contaminación con minas terrestres, artefactos explosivos sin detonar y artefactos explosivos improvisados. Esta condición hace difícil la entrega de ayuda humanitaria en varios departamentos, incluidos el Cauca, Nariño, el Putumayo y Norte de Santander. En este sentido ha habido denuncias de restricciones impuestas por grupos armados ilegales como, el Ejército Revolucionario Antisubversivo de Colombia - ERPAC-, Las Águilas Negras, Los Rastrojos y Los Urabeños en las zonas urbanas de los departamentos de Antioquia, Córdoba y el Valle del Cauca (ONU - Consejo de Seguridad, 2013: web).

El desplazamiento forzado es sin lugar a dudas una de las afectaciones más visibles y significativas en el conflicto colombiano y se incrementa año tras año. En todas sus épocas se ha reportado cómo un alto número de los desplazados internos en el país está conformado por niños y niñas. Para el año 2006, de acuerdo a Cohen (2006) al hablar de características sociodemográficas de los desplazados menciona que "el 23.2\% de la población son niños menores de 7 años" (Cohen, 2006, Pág. 93). Así mismo según la Consultoría para los Derechos humanos y el Desplazamiento - CODHES (2006) citado por Cohen (2006) "alrededor del 50\% del total de las personas en situación de desplazamiento son niños, niñas y adolescentes menores de quince años" (Cohen, 2006, Pág. 93).

En 2012, más de 46.000 personas, de las cuales aproximadamente el 30\% eran niños, sufrieron desplazamiento interno en 18 departamentos, afectando directamente a comunidades rurales, indígenas y afrocolombianas (ONU - Consejo de Seguridad, 2013). Las consecuencias que trae consigo el desplazamiento forzado en niños y niñas, son importantes evidenciándose en miedos, pesadillas, pensamientos repetitivos sobre los hechos dolorosos, el deseo de huir de aquello que recuerda los momentos difíciles pudiendo denominarse estrés postraumático (Vaca \& María, 2009). "En cuanto a desplazamiento forzado, el registro histórico de la Unidad de Atención a Víctimas, a diciembre de 2012, indica que un 26 por ciento de la población desplazada tenía entre 0 y 17 años al momento de tener que dejar sus lugares de origen." (El Colombiano.com, 2013: web).

\subsection{Discursos sobre menores en medios impresos}

El discurso es una categoría relevante en las ciencias sociales desde finales la década de 1990. Tiene múltiples comprensiones y para este estudio es comprendida desde la perspectiva de la psicología social discursiva (İñiguez, 2006 y Sisto, 2012) a partir de la cual se entiende por discurso aquella organización de significados y comprensiones que a través de su empleo construyen la realidad y definen posibilidades para la acción. Comprender el discurso es comprender la acción de la misma forma que aquello que no podrá realizarse por no estar presente en él. Un énfasis en esta comprensión del discurso se centra en el estudio de los efectos que tiene cualquier enunciación, dado que se está haciendo alusión a la coordenadas que orientan la 
acción y por consiguiente las relaciones entre las personas y de ellas con el mundo (Doménech, 1998; Latour, 2008).

El discurso construye versiones sobre el mundo. Sisto (2012) presenta una síntesis clara no sólo de la noción sino también de las funciones que siempre son objeto de análisis en función de las consecuencias, los efectos de los discursos. La función del discurso implica la construcción de versiones lo que se demuestra por la variación del lenguaje. La construcción de eventos a través del lenguaje supone el uso de recursos lingüísticos preexistentes en él mismo. Por su parte la interacción comunitaria a través de diferentes fuentes de significado da cuenta de eventos, concebidos como una realidad más allá de la misma interacción, por lo que el habla cotidiana, en tanto orientada a dar cuenta de eventos, puede ser concebida como una potente constructora de realidad. La variación como característica del discurso emerge de la observación del habla cotidiana y hace referencia a que el lenguaje cambia constantemente de funciones, de acuerdo con las transformaciones de contextos. El lenguaje puede ser usado con una gran variedad de funciones y su uso implica una amplia variedad de consecuencias; un mismo fenómeno puede ser descrito de una gran variedad de maneras, como por ejemplo a través del género periodístico impreso, que hace posible dar cuenta de versiones de cualquier acontecimiento.

Los contenidos del discurso siempre son instituyentes y contribuyen a posicionar versiones acerca del mundo que orientan la acción (Bronwyn \& Harré, 1990: Harré \& van Langenhove, 1999). Es a partir de este proceso de posicionamiento que se trazan relaciones entre las personas y se definen las condiciones en las que se actúa en el mundo, motivo por el cual es importante comprender las categorías presentes en los contenidos discursivos, con indiferencia de la fuente de la que provengan; en todos los casos los discursos construyen realidad y posicionan contenidos referenciales para la acción. Los medios de comunicación masiva son una de las fuentes creadoras, repetidoras y amplificadoras de discurso por lo que se ha prestado especial atención a su dinámica (Bednarek, 2005; Johnson \& Milani, 2010; Matheson, 2005). En este sentido, el estudio del discurso en los medios de comunicación masiva, supone una amplia gama de estrategias de las ciencias sociales que va desde el estudio de la forma noticiosa y su presentación hasta los contenidos e implicaciones ideológicas de ésta. Es importante anotar que el análisis de discurso (Íñiguez, 2006; Parker, 2005; Potter, 1998) no es por definición la única estrategia para acceder a éste, lo cual permite considerar la noción de discurso como nuclear y en un sentido más amplio que la remita a otras estrategias metodológicas de la psicología y las ciencias sociales, incluido el campo disciplinar de la comunicación y el periodismo.

La relación niños-guerra-afectación-medios de comunicación constituye un complejo que se define y se construye a través de discursos específicos. Si bien el complejo nocional no es nuevo, tampoco está ampliamente documentado en los medios académicos, motivo que remite a confirmar la pertinencia de esta investigación. Los estudios se han centrado en los actos violentos que los niños pueden cometer como consecuencia del contacto con discursos presentes en los medios, como ha sido documentado para Sierra Leona (Denov, 2012: web) y Bosnia (Roberts, 2009 "et al"), o en los estudios de Brandl (2011) quien informa acerca de la influencia de los medios de comunicación en el uso de juguetes bélicos por parte de niños. Por su parte Khan (2011) informa acerca de la manera en que el discurso de los medios acerca del trabajo infantil contribuye a construir una política pública que lo prevenga. Estos ejemplos permiten formular al menos dos conclusiones conceptuales. La primera es 
que los trabajos se remiten a temas dispersos de la relación que nos ha interesado, y en segundo lugar, no hay una consideración de las construcciones que los medios hacen de los niños afectados por la guerra a través de los discursos que emplean. Estas conclusiones contrastan con la documentación que confirma la presencia constante de los niños en la guerra (James, 2002; Martínez \& López, 2009), sugiriendo la necesaria ampliación de estudios y ámbitos de análisis.

La investigación sobre los efectos de la guerra en los niños en Colombia está en desarrollo y los trabajos dan cuenta de un interés en este campo. La atención se ha centrado, por ejemplo, en: (a) la manera como los menores de edad son vinculados a los grupos armados irregulares considerándose víctimas del conflicto (AmadorBaquiro, 2010; Carmona "et al", 2010); (b) el análisis de diversos efectos de la guerra en los niños (Vanegas, "et al", 2011); (c) consideraciones políticas emergentes de la presencia de los actores en cuestión en la guerra (Ortega-Jurado, 2012; Ramírez, 2009); (d) la atmósfera sociomoral de menores desvinculados de grupos armados (Estrada, "et al" 2006); o, (e). Los factores psicosociales asociados con Resiliencia en niños afectados por violencia doméstica (Amar, "et al", 2003).

Por su parte, los estudios que relacionan menores afectados por el conflicto político armado colombiano no son abundantes pero comienzan a aparecer. En primer lugar se encuentran aquellos que se refieren la variable psicosocial de influencia de los medios sobre niños en condición de desplazamiento (Rubiano-Daza \& ArgüelloGuzmán, 2010), y en segundo lugar los que se refieren a modelos de autoridad configurados a partir de la exposición a los media (Duarte-Duarte, 2013). La investigación referida a la prensa escrita en Colombia en relación con los niños víctimas del conflicto en Colombia también es escasa (Pardo, 2008; Valencia, 2013), y se trata de proyectos realizados en campos diferentes al de la Psicología Social tal como la relación entre menores, conflicto armado y prensa escrita (Bohórquez, "et al", 2014).

De acuerdo con lo anterior se hizo el planteamiento de esta investigación que indagó por el discurso que dos medios impresos producen en relación con niños, niñas y adolescentes menores de edad que han sido afectados/as por el conflicto político armado en Colombia. El proyecto tiene en cuenta las afectaciones que pueden sufrir los menores en contextos de guerra, y la manera como la prensa se refiere a ellos. La noción de discurso constituye el eje analítico central al considerarlo presente en cada texto de la prensa, escrita o no, con capacidad de agenciar la acción de quienes leen o tienen contacto con la pieza informativa.

\section{Metodología}

La investigación desarrollada fue descriptiva, exploratoria de carácter transversal. Para ello se realizó análisis de contenido para identificar categorías discursivas presentes en las noticias referidas a niños afectados por el conflicto político armado en Colombia, en los dos periódicos impresos de mayor circulación en la ciudad de Bucaramanga y el nor-oriente colombiano.

\subsection{Muestra: unidades de Análisis}

La investigación se hizo con los dos periódicos de mayor circulación en la ciudad de Bucaramanga el nor-oriente colombiano: Vanguardia Liberal y El Frente en el año 
2011. Durante el año circularon un total 519 ejemplares, de los cuales 363, fueron de Vanguardia Liberal y 156 de El Frente. El total la muestra fue de 52 ejemplares por medio, para un total de 104 periódicos, que representa el 20\% del total de periódicos divulgados ese año. Para cada mes se hizo una selección aleatoria según la técnica de semana compuesta de los ejemplares a analizar y las fechas de los ejemplares analizados fueron las siguientes: enero 2-3-11-19-27, febrero 4-12-20-21, marzo 1-9-17-25, abril 2-10-11-19-27, mayo 5-13-21-29-30, junio 7-15-23, julio 1-9-1718-26, agosto 3-11-19-27, septiembre 5-13-21-29, octubre 7-15-23-31, noviembre 1-9-17-25, y diciembre 3-11-12-20-28.

\subsection{Procedimiento}

El estudio asumió las pautas planteadas por Krippendorff, (1997) y se enmarcó en el denominado análisis de tipo semántico, el cual a su vez desarrolla en su procedimiento el análisis de designaciones, el análisis de atribuciones, y el análisis de las aseveraciones. Con el primeo se logró establecer las frecuencias con las que se hace referencia a determinados objeto-sujeto, el segundo otorgó frecuencias a ciertas características de un objeto-sujeto de estudio y el tercero, proporcionó las frecuencias con que ciertos objetos-sujetos son caracterizados de un modo particular.

El estudio revisó la información periodística que publicaron los diarios Vanguardia Liberal y El Frente que hizo referencia a los niños y niñas víctimas del conflicto (unidad de muestreo) y para lograr la identificación de la información se revisaron condiciones de presentación y contenido. La nota informativa (unidad referencial) se clasificó de acuerdo a la categoría géneros periodísticos (unidad de registro) y de ellas se cuantificaron (unidad de enumeración) aspectos como extensión en centímetros y columnas, notas complementarias y ubicación en el cuadernillo y jerarquización. Igual procedimiento se aplica al componente gráfico. Otros componentes que respondieron a las unidades de análisis acordadas fueron: lugar de registro de la información, procedencia, identificación del autor o responsable de la información, tipo y número de fuentes informativas, participación del sujeto (niño, niña) en la información y lugar de ocurrencia de los hechos, fueron unidades físicas y unidades de contexto que se indagaron en los periódicos.

\section{Resultados}

De las 56 notas periodísticas analizadas el 75\% pertenecieron a Vanguardia Liberal y $25 \%$ al El Frente. Dichas informaciones, no están supeditadas únicamente a la relación Estado-subversión en Colombia, sino que involucran a otros actores armados vinculados con el conflicto, tales como paramilitares, bandas criminales, grupos de narcotraficantes y delincuencia común, quienes involucran en sus acciones niños y niñas. En algunas citas se encontró información de niños vinculados con conflictos en otros países, y el contenido discursivo sigue la misma tendencia del caso colombiano.

En los periódicos analizados se encontró que la sección Judicial fue donde se registró el mayor porcentaje de información relacionada con niños, niñas y conflicto armado (38\%). Le siguió la sección Nacional con 27\%, la sección Metropolitana con un $7 \%$, mientras que las secciones regionales incluyeron niños/niñas en el conflicto 
en un 7\%. Las secciones Local, Editorial, Entretenimiento, Deportes y Opinión no dieron cuenta de los niños, niñas y adolescentes en el conflicto.

Con respecto a lugar donde se originó el hecho, el 34\% fue generado en los municipios colombianos, y utilizó en su elaboración testimonios o fuentes informativas de carácter o tipo oficial (47\%) en cabeza de un representante del Gobierno Local y también se utilizó testimonios de fuentes personalizadas (53\%). La voz de los menores no fue tenida en cuenta al momento de redactar las notas informativas.

La categoría discursiva de mayor registro en las notas analizadas fue Maltrato o explotación laboral con 28\%. La segunda con mayor frecuencia fue Menores desplazados y refugiados y utilizados en conflictos armados se registraron en $24 \%$ del total de las notas estudiadas.

Un ejemplo de la categoría Menores desplazados y refugiados y utilizados en conflictos armados fue el registrado en el diario El Frente en una nota que titula "Caen más inocentes por minas antipersonal". En ella se narra la muerte de un erradicador de cultivos ilícitos y las lesiones que les dejó a otras dos personas. Como antecedente la noticia presenta que los niños son las victimas más frecuentes de este fenómeno ya que son los que transitan por los caminos y senderos de los campos. "La última víctima menor, como Elkim Leonardo Pérez, de 13 años de edad, quien hace menos de un mes, el 28 de febrero, activó un artefacto explosivo que le causó la muerte, en el corregimiento Banderas, de San Calixto (Norte de Santander)" (El Frente 2011, 23-03).

Otro ejemplo en esta categoría es la breve-capsula titulada "Falso positivo": "La Fiscalía procesó ayer al exmilitar Jhon Jairo Muñoz por el homicidio de Ober Oyuela Devia, campesino de 15 años que fue reportado como guerrillero muerto en combate con tropas del Ejército en 2006 en el municipio de Mononguete, Caquetá". (Vanguardia Liberal 2011, 09). En este caso la noción con la que se alude al menor es diferente a la nota anterior en donde se presentó como Inocente; en esta ocasión el menor se construye como guerrillero, categoría que contrasta con la definición anterior.

Para el caso de la niña(s) la tipología de mayor registro fue Menores desplazados y refugiados con $37 \%$, seguido de Maltrato o explotación laboral con $31 \%$. Las tipologías Menores desplazados y refugiados y utilizados en conflictos armados se encontró en un $16 \%$ de las notas. Lo registrado en Vanguardia Liberal con el título "Frustraron el secuestro de una joven de 17 años y de sus pequeños hijos", muestra la situación que pasaron por varias horas madre e hijas a quienes intentan secuestrar en el municipio de Florián, Santander: “...Minutos después de que la joven y sus hijos fueron plagiados, cuando transitaban por un sitio conocido como Buenos Aires, en la vereda El Centro, una de las motocicletas, en la que iba un secuestrador, la víctima y su hijo de dos meses, se encontró de frente con la Policía " (Vanguardia Liberal, 2011,05).

Por su parte el periódico El Frente registró cómo a los presuntos autores del secuestro de una menor se les dicta medida de aseguramiento: "Un juez de control de garantías dictó medida de aseguramiento contra tres presuntos integrantes del frente 54 de las FARC, sindicados de ser los autores del secuestro de la pequeña de 10 años Nora Valentina Muñoz". (El Frente, 2011,12).

Con respecto a la categoría Maltrato o explotación laboral, la Agencia de Noticias EFE de la cual toman información los diarios estudiados, denuncia lo ocurrido con las niñas indígenas del país, en particular en la zona selvática del Guaviare. En la 
nota se advierte que se raptan las menores, que según la información, son utilizadas para vender droga y prostituirse. “...Existe una "cacería de niños, explicó el presidente de la ONIC. Luis Evelio Andrade, quien detalló que esa práctica se está registrando especialmente en el departamento selvático del Guaviare. Según Andrade, los que "desaparecen" durante dos o tres días, e incluso a veces no regresan, son en su mayoría niñas indígenas de entre 12 y 14 años, que presuntamente son obligadas por sus captores a prostituirse y a vender droga. También se refirió a esa "caza" Jesús Flores, investigador de la ONIC, para quien el 90\% de los pueblos indígenas está en riesgo de desaparecer por el hambre y la violencia del conflicto armado interno" (Vanguardia Liberal, 2011, 01-03).

De otra parte, en lo que respecta a la situación del niño, niña cuando actúa como victimario, se ubicaron las categorías Desmovilizados (15\%) y Capturados $(85 \%)$. En esta última se identifican tanto el victimario que produce daño o sufrimiento, como el integrante de grupos al margen de la Ley.

En la noticia de abril 27, Vanguardia informa que mediante operativos se capturaron a 29 personas sindicadas de pertenecer a "Los Urabeños". El segundo párrafo inicia: "Entre los detenidos hay dos menores de edad". En el cuarto párrafo se generaliza sobre las acusaciones de las que serán dictaminadas a todos, incluidos los menores. "Estas personas son acusadas de los delitos de homicidio, extorsión y comercialización y tráfico de estupefacientes en la región, por lo que serían indiciados por el delito de concierto para delinquir agravado". (Vanguardia Liberal, 2011, 27-04).

Respecto a la descripción que se hace en la publicación del menor y su familia, los datos obtenidos señalan, que se expuso en el periódico el rostro completo $(10 \%)$, el perfil (3\%) y se distorsionó la imagen (8\%). Igualmente en las notas periodísticas se publicó la edad (23\%), el sexo $(22 \%)$, el nombre $(2 \%)$, lugar de residencia $(19 \%)$ y los delitos de los que fue víctima (18\%) y los cometidos (9\%). En lo relacionado a la familia del niño, niña, solo un 33\% de la información difundió datos, de los cuales sobresalió el registro del nombre de Padre-Madre-Hermanos (13\%), la ubicación geográfica de residencia, (11\%) edades de los familiares (7\%) y la ocupación-labor (2\%).

\section{Discusión y conclusiones}

Las categorías con base en las cuales se construye el discurso de los niños niñas y adolescentes afectados por el conflicto político armado colombiano en medios impresos de una ciudad colombiana confirman los seis tipos de victimización posible que fueron señaladas por ONU — Niños y los Conflictos Armados, (2012). No obstante la singularidad en la manera como se desarrollan y presentan en los dos medios estudiados permite concluir que el discurso en relación con los menores en el conflicto colombiano tiene singularidades.

Se debe destacar que la mayoría de la información relacionada con los menores en el conflicto aparece en secciones judiciales o regionales, en las cuales se relatan acontecimientos que han alterado el devenir cotidiano de las comunidades en las cuales han estado los menores en cualquiera de los roles posibles en los que son construidos. La Sección Judicial con 38\% indica que las notas informativas que relacionan a los niños con el conflicto se consideran de orden público generando la relación menores-delincuencia, menores-víctima, menores-victimario. Dicho en otras palabras, las noticias que construyen realidad en torno a los menores en el 
conflicto se relacionan con acontecimientos abruptos de la violencia en los cuales ellos son parte de los actores en cuestión. En sentido contrario, la ausencia de reflexiones en secciones nacionales o editoriales, especialmente, pone de presente la baja relevancia que los medios de comunicación impresa otorgan al fenómeno de la victimización infantil como consecuencia del conflicto político armado colombiano. La omisión de este tema en secciones editoriales, inquieta en relación con el discurso que los periódicos impresos agencian hacia los lectores y la consecuente comprensión del fenómeno y construcción de realidad vinculada a éste.

¿Cuál es la voz que se refiere a los menores? Es llamativo que los niños, niñas y adolescentes que protagonizan las noticias estudiadas carecen de voz propia para referirse a la experiencia de la cual han sido afectados. Siempre son terceras personas quienes se refieren a la situación de los menores en el conflicto, incluso en pocas ocasiones son los familiares quienes son identificados como fuente. Resalta lo anterior que la voz de los niños y niñas afectadas no es tenida en cuenta por redactores de noticias y llama la atención la nula consulta a dos instituciones del escenario público colombiano como son la Iglesia Católica y la Academia. Las dos entidades han participado de manera directa e indirecta en la transformación de fenómenos sociales y en particular del conflicto armado por lo que también podrían ser fuentes a consultar.

Así, la capacidad e identidad discursiva de los menores afectados por el conflicto es tergiversada por fuentes terciarias que en la mayoría de los casos ni siquiera es la familia. En este mismo sentido la construcción discursiva de los menores en las notas estudiadas sólo tiene en cuenta a su familia en un 33\% y se hace un énfasis mayor en el lugar de procedencia, el sexo y la edad, constituyéndose en categorías estereotípicas. Estas categorías estereotípicas contribuyen en la construcción de un discurso que posteriormente, mediante el uso de los estereotipos, permita enunciar generalizaciones como al afirmar la procedencia, la edad y el sexo del menor. La reunión de estas condiciones de acuerdo con contenidos específicos permitiría la construcción de discursos fácilmente identificables en relación con la presencia de menores en el conflicto bien como afectados o victimarios.

Legalmente la diferenciación de menores afectados-victimarios en el conflicto político armado no es posible. Todas las personas menores de 18 años vinculadas con alguna acción en el marco del conflicto son afectadas de éste. No obstante el tipo de acciones que cometen en el caso de quienes han ejercido la violencia les convierte en causantes de daño a terceros y por consiguiente en un plano estrictamente descriptivo son considerados victimarios. En el contexto de las notas periodísticas analizadas se encuentra diferentes categorías con base en las cuales se construye a los menores de acuerdo con su relación con el conflicto.

En el caso de los menores afectados son construidos con base en dos grandes categorías. La primera de ellas es ética, en la que se definen a partir de lo que supone la minoría de edad o la infancia: la inocencia, lo sano. La segunda de las categorías de se refiere al tipo de afectación: secuestro, homicidio, reclutamiento forzado. Es importante señalar que en ambos casos, el uso de la categoría es descriptiva y no profundiza la experiencia de los menores, sus familiares o allegados. Las notas hacen uso de la noción inocencia, secuestro, homicidio, para describir un hecho y no se trasciende a la experiencia de los actores de la noticia. En otras palabras las categorías discursivas en relación con la afectación de los menores, describen hechos que contribuyen en la construcción del acontecimiento violento pero no la experiencia de los actores de quienes se habla en la nota; se construyen hechos y no identidades, 
historias de vida, que alejan al lector de la experiencia del conflicto, colocándolo de frente a hechos éticamente deplorables.

Sin embargo no todos los menores tenidos en cuenta en las noticias escritas en los diarios estudiados son relatados de la misma manera ni con base en las mismas categorías discursivas. Llama la atención la manera como se construye el discurso en relación con los niños indígenas, en donde la minusvalía y discriminación negativa contribuyen en la construcción de un discurso que podría calificarse de xenófobo. El rapto de niñas que en otras notas es construido a partir de la noción de homicidio o reclutamiento forzado, constituye en las menores indígenas una situación de cacería, consolidando una categoría que deshumaniza a las personas de las cuales se hace alusión. En un contenido retórico que alude a repertorios interpretativos de lo salvaje, no civilizado y distante, se construye a las menores víctimas de rapto, reclutamiento forzado y homicidio como "animales que se cazan", y se marca diferencia en la forma en cómo se habla de ellas en relación con las menores de otras comunidades no indígenas. Además de esta conclusión, las demás condiciones discursivas siguen haciendo parte de estas notas periodísticas en las que se omite la voz en primera persona o familiar, y se redacta en secciones judiciales, nacionales o regionales, por fuera de las columnas de opinión de los periódicos.

La carencia de artículos ubicados en géneros de opinión produce preocupación frente a la imparcialidad pretendida de los medios analizados, dado que su posición puede ser un relevante a la hora de definir política pública o de convocar a la sociedad a acciones en favor de la niñez. Cabe destacar que si bien es cierto la imparcialidad de los medios es una de sus principales características y signos visibles de seriedad, también es verdad que un medio de comunicación debe tener apuestas que se concreten en la posibilidad de la construcción social, con mayor énfasis tratándose de la población menor de 18 años. Una construcción que equilibre las voces de los protagonistas de las noticias así como su presencia en las diferentes secciones de los medios impresos supone una necesidad.

La actual condición en la que se construye el discurso de menores vinculados al conflicto orienta al lector a acciones limitadas. El desconocimiento de la experiencia de vida de los menores, el énfasis en hechos de violencia en los que se encuentran involucrados, la definición de categorías sociodemográficas para referirse a los menores, la ausencia de un posicionamiento discursivo en relación con la afectación de los menores, y los sesgos adjudicados en función de las poblaciones de origen son las principales características de las categorías discursivas con que los medios de comunicación impresos se refieren a niños niñas y adolescentes afectados por el conflicto político armado colombiano. Dichas categorías imponen limitaciones efectivas al lector para que se posicione de manera proactiva en la transformación del conflicto debido al énfasis colocado en los hechos de la violencia y en las historias de las personas inmersas en ellas. Esta conclusión ratifica la necesidad del trabajo en pro de la memoria histórica que tenga como eje central las historias de las personas afectadas en primera persona como ya sucede pero sin la amplificación suficiente que ni siquiera los medios recogen de manera decidida.

El posicionamiento discursivo que se ha encontrado en esta investigación define condiciones de relación específicas entre las personas que leen la prensa y aquellas con las cuales se relacionan, con quienes los contenidos son compartidos. Es innegable el papel que cumplen los medios impresos en la generación de agenda pública y por consiguiente construcción de realidad y condiciones de posibilidad para las 
relaciones. De esta conclusión emerge una pregunta que no ha sido objeto de este estudio pero que marca una línea de continuidad: ¿cuáles son los efectos relacionales del énfasis en los actos de violencia más que en las historias de sus protagonistas?, protagonistas que se podrían considerar afectados, victimarios y sociedad civil al mismo tiempo.

A partir del Análisis de Contenido de Tipo Semántico, se logró entender cómo los niños y niñas víctimas del conflicto armado en Colombia fueron construidos discursivamente en los periódicos locales, con qué frecuencia se hizo referencia a ellos, en qué tipo de hechos se involucraron y qué características de los niños y niñas víctimas del conflicto se registraron durante el lapso analizado, pero también lo que no se dijo acerca de sus vidas.

Se sugiere la continuación de indagaciones sistemáticas dirigidas a las audiencias (lectores) en busca de conocer las categorías discursivas que guardan relación con aquellas presentadas en los diferentes medios. También quedan inquietudes en relación con ¿qué posicionamiento tienen los niños y niñas acerca del conflicto armado en relación y la forma en que son presentados por la prensa escrita, o de otra índole?

El desarrollo de la investigación confirma la necesidad de realizar este tipo de pesquisas, cada vez con mayor divulgación e impacto académico y político en los medios. Como fue señalado en la introducción del estudio, son escasos los trabajos que en el mundo se hacen bajo la combinatoria de conflictos armados, menores, psicología social y medios de comunicación. Atender el efecto del contenido discursivo de los medios es relevante en el contexto de la guerra por el tipo de relaciones capaz de crear. Si bien puede transformar la guerra en paz, también puede mantener la guerra con la guerra a través de la manera como habla de ella. Cabe concluir en este sentido con las palabras de Ignacio Martín Baró (2003: 310) quien señaló: "como psicólogos, no podemos entonces darnos por satisfechos con atender las situaciones post-traumáticas. Esto es necesario y particularmente urgente con los niños. Pero el problema de fondo no está en los individuos, sino en las relaciones traumatogénicas propias de un sistema opresor que ha desembocado en una situación de guerra", en donde algunos medios a través de sus discursos hacen parte de ese sistema.

\section{Referencias bibliográficas}

Agencia PANDI (2008): La Huella de la niñez en la prensa colombiana. Bogotá, TC Impresores S.A.

Amador-Baquiro, Juan Carlos (2010): "El intersticio de víctima victimario: un análisis de los procesos de subjetivación de cuatro desvinculados de grupos armados en Colombia". Universitas Humanistica, 69, 163-184. Bogotá, Revistas Javerianas.

Amar, José Juan; Kotliarenko, María Angélica y Abello, Raimundo (2003): "Factores psicosociales asociados con la resiliencia en niños colombianos víctimas de violencia familiar". Investigación y Desarrollo, 11 (1), 162-197. Barranquilla, Universidad del Norte.

Amnistía Internacional (2004): "Vidas rotas. Crímenes contra mujeres en situaciones de conflicto. No más violencia contra las mujeres", en Amnesty International Publications. http://www. amnesty.org/es/library/asset/ACT77/075/2004/es/9dbc9ef3-d57f-11dd-bb24-1fb85fe8fa05/ act770752004es.html\#3.Cap\%C3\%ADtulo\%203.\%20La\%20violaci\%C3\%B3n\%20 como\%20arma\%20de\%20guerra|outline. [Consulta: 10 de febrero de 2014] 
Bednareck, Mónica (2006): Evaluation in media discourse: analysis of a newspaper corpus. London, Continuum.

Bohórquez, Giovanni; Bohórquez, Ledis; y Puyana, Beatriz Inés (2014): "Prensa local, menores y conflicto armado colombiano. Análisis y efectos en la agenda pública". Dixi, 16 (20), 45-58. Bogotá, Universidad Cooperativa de Colombia.

Brandl, Michael (2011): "Children's play and militarty toys today". Psychological Science and Education, 2, 26-29. Moscow, State University of Psychology and Education.

Bronwyn, Davies \& Harré, Rom. (1990): "Positioning: the discursive production of selves". Journal of Theory and Social Behaviour, 1, 43-63. London, Wiley.

Carmona, Jaime Alberto; Moreno, Florentino Martín; Tobón, John Felipe (2010): “Child soldiers in Colombia. Five Views". Universitas Psychologica, 11 (3), 755-768. Bogotá, Revistas Javerianas.

COALICO [Coalición contra la vinculación de niños, niñas y jóvenes al conflicto armado en Colombia] (2013): Boletín de Monitoreo, 11 Diciembre:

www.coalico.org:http://www.coalico.org/images/stories/bonca_11.pdf [Consulta: 25 de marzo de 2014]

Cohen, Carolina (2006): “Atención a población en situación de desplazamiento y alternativas para la estabilización socioeconómica desde la generación de ingresos". Pensamiento Psicológico, 7 (2), 89-112. Pontificia Universidad Javeriana.

Correa, Mary (2008): "El Lenguaje de los medios que intensifica el conflicto armado colombiano". Reflexión Política, 19, Año 10, 106-113. Publicaciones UNAB.

Denov, Myriam (2012): “Child soldiers and iconography: portrayals and (mis)representations". Children and Society, 26, 2010, 280-292. DOI:10.1111/j.1099-0860.2010.00347.x.

Doménech, Miquel (1998): "El problema de lo "social" en psicología. Algunas consideraciones desde la sociología del conocimiento científico". Revista Anthropos, 177, 35-39. Barcelona, Editorial Anthropos.

Duarte-Duarte, Jakeline (2013): “Infancias contemporáneas, medios y autoridad”. Revista Latinoamericana de Ciencias Sociales, Infancia y Juventud, 11 (2), 461-472. Manizales, Universidad de Manizales.

El colombiano.com (2013): "ICBF reveló cifras sobre victimización de niños por conflicto", en El Colombiano.com:

http://www.elcolombiano.com/icbf_revelo_cifras_sobre_victimizacion_de_ninos_por_ conflicto-EEEC_237030. [Consulta: 18 de abril de 2015]

Estrada, Ángela María; González, Constanza; Diazgranados, Silvia; y Toro, Manuel (2006): "Atmósfera socioemocional y atención de menores desvinculados del conflicto armado en Colombia". Revista Infancia, Adolescencia y Familia, 1 (002), 223-246. Bogotá, Asociación Colombiana para el Avance de las Ciencias del Comportamiento.

Harré, Rom \& Langenhove, Van (1999): Positioning theory. Oxford, Balckwell Publishers. Íñiguez, Lupicinio (2006): Análisis del discurso. Manual para las ciencias sociales. Barcelona, UOC.

James, Marten (2002): Children and war. A historical antology. New York, New York University Press.

Johnson, Sally \& Milani, Tommaso (Eds. 2010): Language, ideologies and media discourse. Texts, practices and politics. London, Continuum.

Khan, Ali (2010): "Discourses on chilhood: Policy-Making with regard to child labour in the context of competing, cultural and economic perceptions". History and Anthropology, 21 (2), 101-119. DOI: 10.1080/02757201003730574. 
Krippendorff, Klaus (1997): Metodología de Análisis de Contenido, Teoría y Práctica. Barcelona, Paidós.

Latour, Bruno (2005): Reensamblar lo social. Una introducción a la teoría del actor-red. Buenos Aires, Manantial.

Martín-Baró, Ignacio (2006): Poder, ideología y violencia. Madrid, Trotta.

Martínez, Noemí y López, Marián (2009): Reinventar la vida. El arte como terapia. Madrid, Eneida.

Matheson, Donald (2005): Media discourses: analyzing media texts. Glasgow, Open University Press.

ONU [Organización de Naciones Unidas - Consejo de Seguridad] (2013): Los niños y los conflictos armados. Informe del Secretario General (A/67/845-S/2013/245). En Naciones Unidas http://reliefweb.int/sites/reliefweb.int/files/resources/N1331170.pdf. [Consulta: 24 de marzo de 2015]

ONU [Organización de Naciones Unidas — Consejo de Seguridad] (2012): "Informe del Secretario General sobre los niños y el conflicto armado en Colombia (S/2012/171)", en Naciones Unidas Consejo de seguridad, en: http://www.acnur.org/t3/fileadmin/scripts/doc.php?file=t3/uploads/media/Informe del_Secretario_General_sobre_los_ninos_y_el_conflicto_armado_en_Colombia__S_2012_17 [Consulta: 20 de marzo de 2015]

Ortega-Jurado, Mario Fernando (2012): "Protección de la infancia y la adolescencia en asuntos de derecho internacional humanitario en Colombia". Revista Colombiana de Derecho Internacional, 20, 13-46. Bogotá, Facultad de Ciencias Jurídicas, Pontificia Universidad Javeriana.

Pardo, Clara Cecilia (2008): "La prensa, ventana de los objetivos de desarrollo del milenio: mortalidad infantil, salud materna, VIH/Sida en Colombia”. Palabra Clave, 11 (2), 165177. Bogotá, Universidad de la Sabana.

Parker, Ian (2005): Qualitative psychology. Introducing radical research. Glasgow, Open University Press.

Potter, Jonathan (1998): La representación de la realidad. Discurso, retórica y representación social. Barcelona, Paidós.

Ramírez, Patricia (2009): “Ciudadanías negadas: victimización histórica, reparación y (re) integración para mujeres y niñas en Colombia. El desafío de zurcir las telas rotas”. Reflexión Política, 11 (21), 92-107. Bucaramanga, Universidad Autónoma de Bucaramanga.

Roberts, Ron; Beciarevic, Majda; and Baker, Tracy (2009): "2Children's understanding of the war in Irak: views from Britain and Bosnia". International Journal of Children's Spirituality, 1, 17-30. IJCS.

Rubiano-Daza, Henry y Argüello-Guzmán, Luis Alfonso (2010): "Recepción televisiva de la audiencia infantil con características de desplazamiento forzado". Palabra Clave, 13 (2), 307-322. Bogotá, Universidad de la Sabana.

Sisto, Vicente (2012): “Análisis de discurso y psicología. A veinte años de la revolución discursiva". Revista de Psicología, 1 (21), 185-208. Departamento de Psicología, Universidad de Chile.

UNICEF y CECODAP (2008): La Niñez y la adolescencia en los medios: ¿Cuándo si y cuándo no? Caracas, Ediciones El Papagayo - Mundo Gráfico.

UNICEF (2014): “2014: declarado año devastador para la infancia, 15 millones de niños atrapados en graves conflictos". En UNICEF Actualidad: http://www.unicef.es/actualidad-documentacion/noticias/2014-declarado-anodevastador-para-la-infancia-15-millones-de. [Consulta: 18 de abril de 2015] 
Vaca, Patricia y Rodriguez, María (2009): "Responsabilidad social de la psicología frente a la violencia”. Pensamiento Psicológico, 13 (6), 87-96. Pontificia Universidad Javeriana.

Valencia, Víctor Hugo (2013): "Pornografías de los cuerpos (masculinos/femeninos). Una mirada desde la prensa escrita sensacionalista a los jóvenes excluidos de Cali, Colombia". Signo y Pensamiento, 32 (62), 114-129. Cali, Pontificia Universidad Javeriana.

Vanegas, Julián Alberto; Bolívar, Carlos; y Camacho, Leidy Bibiana (2011): "Significado del desplazamiento forzado por conflicto armado para niños y niñas". Fundamentos en Humanidades, Vol. XII, 24, 163-189. Disponible en: http://www.redalyc.org/articulo. oa? id=18426920007.

Ledis Bohórquez Farfán es filósofa, magister en Educación y Desarrollo Humano, y Profesora asociada e investigadora en el Departamento de Formación Humanística de la Universidad Pontificia Bolivariana, Seccional Bucaramanga (Colombia). Integrante del Grupo interdisciplinario de estudios sobre Cultura, Derechos Humanos y Muerte. Líder del Semillero de investigación en Derechos Humanos. Tiene diversas investigaciones en áreas relacionadas con la ética, ciudadanía y respeto y garantía de los derechos humanos.

Giovanni Bohórquez-Pereira es periodista y Profesor asociado de la Facultad de Comunicación Social de la Universidad Pontificia Bolivariana, Seccional Bucaramanga (Colombia). Integrante de Grupo de Investigación Comunicación Periodismo y Política, COMPPA. Magíster en Ciencias Políticas de la Pontificia Universidad Javeriana, Seccional Cali. Investigador en Opinión Pública, Comunicación y Sociedad. Coordinador del Semillero de Investigación U’wa Werjayá.

Nelson Molina Valencia es doctor en Psicología Social, Subdirector de Investigaciones y Postgrados del Instituto de Psicología de la Universidad del Valle, Colombia, y miembro del Grupo de Investigación Lenguaje, Cognición y Educación. Lidera la línea de investigación en Narrativas, Subjetividad y Construcción Social. 